\title{
Towards a Multivariable Model for Controlling the Depth of Anaesthesia using Propofol and Remifentanil
}

\author{
Clara M. Ionescu*, Ioana Nascu**, Robin De Keyser* \\ *Ghent University, Faculty of Engineering and Architecture, Department of Electrical energy, Systems and Automation, \\ Technologiepark 913, B9052 Gent-Zwijnaarde, Belgium (Tel: 32-9-2645608; e-mail: ClaraMihaela.Ionescu@UGent.be). \\ ** Technical University of Cluj-Napoca, Dept. Automation, Cluj-Napoca, Romania
}

\begin{abstract}
In this paper is presented a brief state of art regarding the multivariable formulation for controlling the depth of anaesthesia by means of two intravenously administrated drugs, i.e. Propofol and Remifentanil. In a feasibility study of determining a suitable variable to quantify analgesia levels in patients undergoing cardiac surgery, the Bispectral index and an electromyogram-based surrogate variable are proposed as the controlled variables. The study is carried on in the context of implementing a multivariable predictive control algorithm. The simulation results show that such a paradigm is feasible, although it does not guarantee perfect knowledge of the analgesia level - in other words, the variable is not validated against typical evaluations of the pain levels (eg. clinical scores).
\end{abstract}

Keywords: anaesthesia, analgesia, predictive control, multivariable control.

\section{INTRODUCTION}

General anaesthesia plays an important role in surgery and intensive care unit (ICU) and requires critical assessment of induced quantities of drugs into the patient (Dumont et al, 2009). It is characterized by unconsciousness through the action of anaesthetics, but also by loss of the ability to perceive pain through the action of analgesics. Analgesics block the sensation of pain; the hypnotics produce unconsciousness, while the muscle relaxants prevent unwanted movement of muscle tone. The relationship between the hypnotic drug, Propofol, administered during general anaesthesia, and BIS (a signal derived from the electroencephalogram used to assess the level of consciousness during anaesthesia) is widely documented and several studies regarding the interaction model of Propofol and Remifentanil can be found in the literature (Morley et al, 2000; Struys et al, 2003; Absalom et al, 2011).

When inducing and maintaining anaesthesia, anaesthesiologists select initial doses based on a variety of considerations, they observe the results, and then make adjustments based on several factors, at irregularly varying intervals. In control engineering terminology, this constitutes a closed loop control system, due to the feedback present in the observations and interventions of the anaesthesiologist. The closed-loop control system is characterized by special feature: i) it has a human controller in the loop, and ii) the control actions are intermittent and irregular in time due to the human controller.

The purpose of computer-controlled closed-loop systems is to formalize the process of observation and intervention as to provide better and more accurate control. Such systems use a near continuous signal of drug effect, calculate the error between the observed value and the specified value (selected by the medical staff), and use this error in an algorithm to make frequent and regular adjustments to drug administration rates. Moreover, some computer-control systems try to predict the future drug effect to produce the optimal convergence to the desired result (Ionescu et al, 2008; Absalom et al, 2011).

In order to have an accurate feedback control, one or more real-time representative measures of the system's state should be available. Ideally, the control actuators or process inputs should, with minimal or known delay, cause predictable, linear changes in the process. In practice, drug administration is an asymmetrical process: we can actively infuse but cannot actively remove the drug from the patient. Because the relationship between dose and plasma concentration is so complex, target-controlled infusion (TCI) systems are a logical choice of control actuator, so that the control input is a target concentration rather than an infusion rate (Kazama et al, 1998; Struys et al, 2003; Absalom et al, 2011). Many assumptions underpin the pharmacokinetic models used in TCI systems, the predictive accuracy of current models is imperfect, and the choice of model for Propofol is often controversial (Absalom et al, 2009).

This paper presents results based on the use of Propofol and Remifentanil as anaesthetic, respectively analgesic drugs to regulate the depth of anaesthesia (DOA). A first attempt is made to provide a variable for the effect of Remifentanil drug infusion on the analgesic state of the patient. This is then tested in a closed loop control simulation by means of applying model based predictive control algorithm. Although the simulation results are not validated against external clinical scores, the paper can serve as an indicator of the challenges one needs to tackle when building a fully multivariable model for automated regulation of anaesthesia and analgesia. 
The paper is organized as follows: the next section introduces briefly the state of art for modelling anaesthesia and analgesia in a multivariable context. Section 3 describes the derivation of the output variable to measure the effect of Remifentanil infusions. Section 4 presents the simulation setup, followed by the simulation results and discussion. Finally, a conclusion section summarizes the main outcome of this paper and points to some further directions of research.

\section{THE MULTIVARIABLE PARADIGM OF AUTOMATED DOA}

For measuring the hypnotic component of anaesthesia, various indexes are present, mostly computerized from the spontaneous or evoked electroencephalogram (EEG) (Absalom et al, 2011). The Bispectral index (BIS) is a single composite measure derived from the spontaneous EEG and has been proven to have a high sensitivity and specificity to measure anaesthetic drug effect (Morley et al, 2000; Struys et al, 2003). BIS is now recognized as one of the reference measures of DOA for closed loop control purposes (Ionescu et al, 2008; Absalom et al 2011; Liu et al, 2011). The singular control of the Bispectral index by means of Propofol drug infusion using computer-assisted DOA has been already established in the literature as advantageous to targetcontrolled infusion (open loop) (Struys et al, 2003; Ionescu et al, 2008; Dumont et al 2009; Yelneedi et al 2009). Hence, this paper will focus on the multivariable paradigm, which includes the effect of opioids (i.e. analgesic drugs).

In contrast to cerebral drug effect produced by hypnotics, an accurate measure for analgesia is still lacking. Opioids such as fentanyl, alfentanyl and Remifentanil are known to have synergistic effect on Propofol (Milne et al, 2003; Kazama et al, 1998; Simanski et al, 2009). Since general anaesthesia is clinically defined as the balance between hypnosis, analgesia and paralysis, it is interesting to study the effect of drug interaction (Milne et al, 2003). It has already been shown that neuromuscular blockade (i.e. the paralysis component of general anaesthesia) is not inter-related to the hypnotic and analgesic components (i.e. no drug interaction) (Da Silva et al, 2011; Simanski et al, 2009). On the other hand, it has been shown that the use of Remifentanil in regulated DOA has a sparing effect on Propofol infusion rates, hence with much less over-dosage occurrences (Milner et al, 2003). The challenge, however, is that these combinatorial effects are varying from one patient to another - interpatient variability - as well as varying within the same patient - intrapatient variability. Often the anaesthetists use a certain drug rate for a long period of time (tens of minutes) during similar surgical procedures, especially in countries where computer assisted DOA is not available. This leads to either under- or overdosage in the patient, both having undesired effects. It was also found that the concentrations for Propofol for which the patient became awake were increasing with the duration of drug administration, showing the potential for hysteresis (Kazama et al, 1998), thus more challenging from control point-of-view.

While feedback monitoring devices and methods are already available on the market for the depth of hypnosis (eg.
Bispectral Index BIS, Auditory Evoked Potential, WAV), there exists no "pain sensor" which measures analgesia directly (Absalom et al, 2011; Zikov et al, 2006). However, there are several systems for analgesia control reported in the literature. Most of them use a surrogate of variables to derive a fuzzy-expert system, which may assist the clinical nurse in determining the optimal analgesic drug rate. A fuzzy analgesia control system for induction, maintenance and recovery has been reported in (Schubert et al, 2008), using three variables: heart rate, mean arterial pressure and an index derived from a (modified) standard deviation of the RR-intervals in the electrocardiogram format. Furthermore, a control system, which minimizes the risks associated with delivery of respiratory depressants to spontaneously breathing patients during medical procedures, has been proposed in (Caruso et al, 2009). This has been based on modelling the respiratory depressant effects of Remifentanil by means of pharmacokinetic (PK)-pharmacodynamic (PD) models in (Caruso et al, 2009) provided the transcutaneous monitoring of partial pressure of carbon dioxide in tidal breathing. However, this strategy may fail in the event of mechanical ventilation, which alters the nominal depressant effect.

A combination of rule-based controller for Remifentanil infusion, based on measurements of mean arterial pressure, heart rate and systolic arterial pressure, and a fuzzy-PI controller for Propofol infusion, based on pre-determined DOA levels, is proposed in (Mahfouf et al, 2005). A predictive controller with isoflurane and alfentanil is given in (Yelneedi et al, 2010) controlling well four variables: bispectral index, mean arterial pressure, end tidal concentration and alfetanil concentration. However, from a control point of view, the control problem is ill-posed, since there are two manipulated variables to control four variables.

Finally, another approach to use cardio-respiratory surrogate variables to measure the level of analgesia led to the development of the analgoscore, which proved to be successful in a manifold of surgical interventions (Hemmerling et al, 2007). Unfortunately, no tests are performed on patients undergoing cardiac surgery, where these surrogate measures coming from the cardio-respiratory variables are obviously biased.

\section{PROPOSED MULTIVARIABLE CONTEXT}

\subsection{Patient Models for Propofol and Remifentanil}

In order to investigate the multivariable formulation from figure 1, the pharmacokinetic (PK) models for Propofol and Remifentanil are required. Additionally, the interaction model represented in figure 1 by the Hill block takes into account the synergistic effect of these two drugs on the output variable, the Bispectral index (BIS). In figure 2 the pharmacokinetic (PK) - pharmacodynamic (PD) blocks denote compartmental models. The PK-PD models most commonly used for Propofol and Remifentanil are the $4^{\text {th }}$ order compartmental models described by Schnider (Schnider et al, 1999) and Minto (Minto et al, 1997a,b) respectively.

The PK-PD models are represented by the following equations: 


$$
\begin{aligned}
& \dot{x}_{1}(t)=-\left[k_{10}+k_{12}+k_{13}\right] x_{1}(t)+k_{21} x_{2}(t)+k_{31} x_{3}(t)+\frac{u(t)}{V_{1}} \\
& \dot{x}_{2}(t)=k_{12} \cdot x_{1}(t)-k_{21} \cdot x_{2}(t) \\
& \dot{x}_{3}(t)=k_{13} \cdot x_{1}(t)-k_{31} \cdot x_{3}(t) \\
& \dot{x}_{e}(t)=-k_{e 0} \cdot x_{e}(t)+k_{1 e} \cdot x_{1}(t)
\end{aligned}
$$

where $x_{1}[\mathrm{mg} / \mathrm{ml}]$ denotes the drug concentration in the central compartment. The peripheral compartments 2 and 3 model the drug exchange of the blood with well and poorly diffused body tissues. The masses of drug in fast and slow equilibrating peripheral compartments are denoted by $x_{2}$ and $x_{3}$, respectively. The parameters $k_{j i}$, for $i \neq j$, denote the drug transfer frequency from the $j^{\text {th }}$ to the $i^{\text {th }}$ compartment and $u(t)[\mathrm{mg} / \mathrm{s}]$ is the infusion rate of the anaesthetic drug into the central compartment. The parameters $k_{i j}$ of the PK models depend on age, weight, height and gender and can be calculated for Propofol:

$$
\begin{aligned}
V_{1}= & 4.27[l] ; V_{3}=2.38[l] \\
V_{2}= & 18.9-0.391 \cdot(\text { age }-53)[l] \\
C_{l 1}= & 1.89+0.0456(\text { weight }-77)-0.0681(l b m-59)+ \\
& +0.0264(\text { height }-177)[l / \mathrm{min}] \\
C_{l 2}= & 1.29-0.024(\text { age }-53)[l / \mathrm{min}] \\
C_{l 3}= & 0.836[l / \mathrm{min}] \\
k_{10}= & \frac{C_{l 1}}{V_{1}}\left[\min ^{-1}\right], k_{12}=\frac{C_{l 2}}{V_{1}}\left[\min ^{-1}\right], k_{13}=\frac{C_{l 3}}{V_{1}}\left[\min ^{-1}\right] \\
k_{21}= & \frac{C_{l 2}}{V_{2}}\left[\min ^{-1}\right], k_{31}=\frac{C_{l 3}}{V_{3}}\left[\min ^{-1}\right]
\end{aligned}
$$

where $C_{l 1}$ is the rate at which the drug is cleared from the body, and $C_{l 2}$ and $C_{l 3}$ are the rates at which the drug is removed from the central compartment to the other two compartments by distribution.

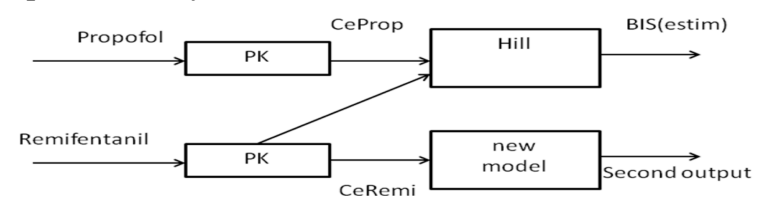

Fig. 1. Diagram of the proposed multivariable formulation. New Model and Second Output are not determined yet.

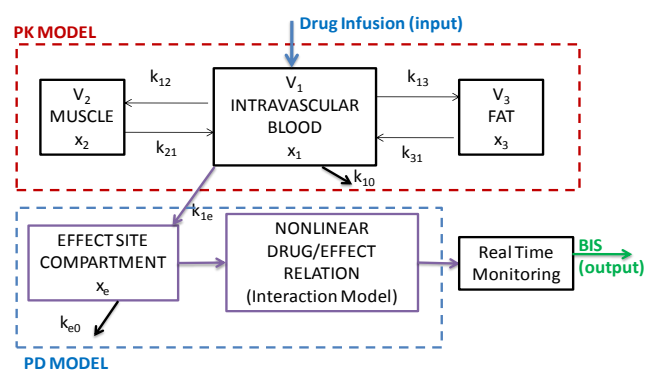

Fig. 2: Compartmental model of the patient, where PK denotes the pharmacokinetic model and PD denotes the pharmacodynamic model.
Similarly, for Remifentanil:

$V_{1}=5.1-0.0201($ age -40$)+0.072(\mathrm{lbm}-55)[l]$

$V_{2}=9.82-0.0811($ age -40$)+0.108(l b m-55)[l], \quad V_{3}=5.42[l]$

$C_{l 1}=2.6-0.0162($ age -40$)+0.0191(l b m-55)[l / \mathrm{min}]$

$C_{l 2}=2.05-0.0301($ age -40$)[l / \mathrm{min}]$

$C_{l 3}=0.076-0.00113($ age -40$)[l / \mathrm{min}]$

$k_{e 0}=0.595-0.007($ age -40$)\left[\mathrm{min}^{-1}\right]$

$k_{10}=\frac{C_{l 1}}{V_{1}}\left[\min ^{-1}\right], k_{12}=\frac{C_{l 2}}{V_{1}}\left[\min ^{-1}\right], k_{13}=\frac{C_{l 3}}{V_{1}}\left[\min ^{-1}\right]$

$k_{21}=\frac{C_{l 2}}{V_{2}}\left[\min ^{-1}\right], k_{31}=\frac{C_{l 3}}{V_{3}}\left[\min ^{-1}\right]$. The lean body mass

(lbm) for men and women has the following expressions:

$1.1 \cdot$ weight $-128 \cdot \frac{\text { weight }^{2}}{\text { height }^{2}}$ and $1.07 \cdot$ weight $-148 \cdot \frac{\text { weight }^{2}}{\text { height }^{2}}$,

respectively, with weight $(\mathrm{kg})$ and height $(\mathrm{cm})$.

An additional hypothetical effect compartment was proposed to represent the lag between drug plasma concentration and drug response. The concentration of drug in this compartment is represented by $x_{e}$. The drug transfer frequency from the central compartment to the effect sitecompartment is equal to the frequency of drug removal from the effect-site compartment: $k_{e 0}=k_{1 e}=0.456\left[\mathrm{~min}^{-1}\right]$. The equation is often referred as the effect-site compartment concentration. When considering the effect of two drugs, the Hill curve becomes a surface, whose parameters represent the synergistic effect of both Propofol and Remifentanil effect site compartment concentrations. The concentration-response relations of the two drugs can be described by a normalized relation:

$$
B I S(t)=E_{0}-E_{\max }(\theta) \cdot \frac{\left(\frac{U_{\operatorname{Pr} o p}(t)+U_{\operatorname{Re} m}(t)}{U_{50}(\theta)}\right)^{\gamma(\theta)}}{1+\left(\frac{U_{\operatorname{Pr} o p}(t)+U_{\operatorname{Re} m}(t)}{U_{50}(\theta)}\right)^{\gamma(\theta)}}
$$

where: $U_{\operatorname{Pr} o p}(t)+U_{\operatorname{Re} m}(t)$ is the combined drug concentration; $\gamma(\theta)$ is the steepness of the concentration-response relation at ratio $\theta ; U_{50}(\theta)$ is the number of units $(U)$ associated with $50 \%$ of maximum effect at ratio $\theta ; E_{\max }(\theta)$ is the maximum possible drug effect at ratio $\theta$ (Minto et al, 2000), with the effect-site concentrations $C_{e \operatorname{Pr} o p}(t)$ and $C_{e \operatorname{Re} m}(t)$ normalized to their respective potencies $C_{50, \operatorname{Pr} o p}$ and $C_{50, \operatorname{Re} m}$ described by:

$$
U_{\operatorname{Pr} o p}(t)=\frac{C_{e \operatorname{Pr} o p}(t)}{C_{50, \operatorname{Pr} o p}} ; U_{\operatorname{Re} m}(t)=\frac{C_{e \operatorname{Re} m}(t)}{C_{50, \operatorname{Re} m}}
$$

and the ratio of the interacting drugs expressed by:

$$
\theta(t)=\frac{U_{\operatorname{Pr} o p}(t)}{U_{\operatorname{Re} m}(t)+U_{\operatorname{Pr} o p}(t)}
$$

In this formulation, $\theta$ represents the concentration ratio of the new combined drug and ranges from 0 (Remifentanil only) to 
1 (Propofol only). According to (Minto et al, 1997a) $E_{\max }(\theta)$ and $E_{0}$ are set to 100 and $U_{50}(\theta)$ can be expressed by a quadratic polynomial:

$$
U_{50}(\theta)=1-\beta \cdot \theta+\beta \cdot \theta^{2}
$$

The unknown coefficient $\beta$ can be estimated from the patient data. Since the interaction between the two drugs is supraadditive (the effect of the two drugs combined is higher than the sum of each separate effect), $\beta$ should be a positive number. This means that $U_{50}(\theta)$ is lower than 1 for any value of $\theta$ between 0 and 1 . To simulate the combined effect of Propofol and Remifentanil using the nonlinear expression from (2), the following values have been assigned (Nascu et al, 2011):

$$
\beta=0.22 ; \gamma(\theta)=0.9 ; C_{50, \text { Prop }}=3.1 ; C_{50, \text { Rem }}=34
$$

\subsection{Proposed Model for the Remifentanil Effect}

There have been several attempts to quantify the effect of Remifentanil on the analgesia level during surgery and intensive care. Some of these are summarized below.

Derived electroencephalogram measures: if we increase the level of Remifentanil the Cortical Input - a measure of the magnitude of cortical input - will significantly decrease. For quantifying the Propofol effect, one can use the Cortical State - a measure of the responsiveness of cortex - which is statistically independent of variations in the effect site Reminfentanil levels (Liley et al, 2010).

Respiratory effect: Remifentanil is a potent ventilatory depressant. Simulations demonstrated that Remifentanil concentrations well tolerated in the steady state will cause a clinically significant hypoventilation following bolus administration, confirming the acute risk of bolus administration of fast-acting opioids in spontaneously breathing patients (Caruso et al, 2009; Pattinson et al, 2009).

Haemodynamic Effects: Remifentanil induces a dosedependent decrease in heart rate, arterial blood pressure and cardiac output consistent with $\mu$-opioid agonism (Caruso et al, 2009; Mahfouf et al, 2005).

Central Nervous System: Remifentanil induces dosedependent changes in relative cerebral blood flow in areas involved in pain processing. Under Remifentanil/N2O anaesthesia, the global cerebral blood flow is reduced (Hemmerling et al, 2007). As a consequence, intracranial pressure is reduced and autoregulation is preserved.

BIS derivative: our prior studies (unpublished) indicated that this signal is more sensitive to artefacts and it responds faster than the BIS signal. This suggests further that if this signal is used for feedback information, the control might have overdosing effects.

Electromyography is a technique for evaluating and recording the activation signal of muscles. An electromyograph detects the electrical potential generated by muscle cells when these cells contract, and also when the cells are at rest. In this study, the relationship between Remifentanil effect-site concentration $\left(C_{\text {eRemi }}\right)$ and the EMG is determined. For this, we make use of an illustrative signal measurement from one virtual patient undergoing general anaesthesia during intensive care. A scaled variable is proposed:

$$
M=\frac{100 \cdot C_{e \mathrm{Remi}}}{E M G}
$$

thus $M$ is determined as a function of the concentration of Remifentanil and the EMG signals. The concentration of Remifentanil versus the new value $M$ is represented in the following figure for one patient in figure 3. With a green dashed line we have represented the relation between the concentration of Remifentanil and $M$ using data measured during ICU and with a blue solid line we have the linear approximation of this relation. This linear approximation has the following formula:

$$
M=3.4 \times C_{e \text { Remi }}+0.0063
$$

Using this equation and equation (7) we can determine a relationship for EMG as a function of concentration of Remifentanil:

$$
E M G=\frac{100 \cdot C_{e \text { Remi }}}{3.4 \times C_{e \text { Remi }}+0.0063}
$$

Figure 4 is used to validate the model for $M$. With a green line we have $M$ calculated using the measured data of EMG and $C_{\text {eRemi }}$ and with a blue line we have $M$ calculated using the linear approximation.

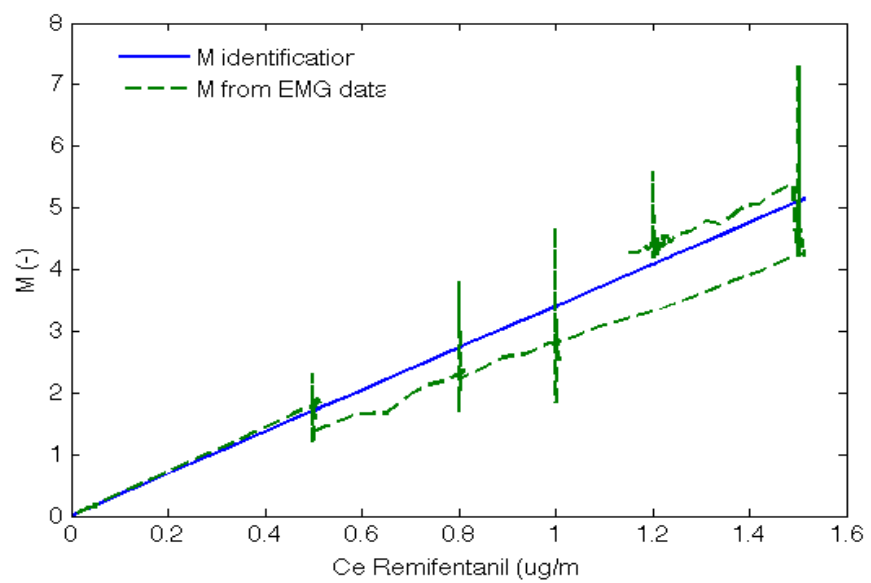

Fig. 3.Representation of $C_{e \text { Remi }}$ versus $M$ and the linear approximation for the virtual patient

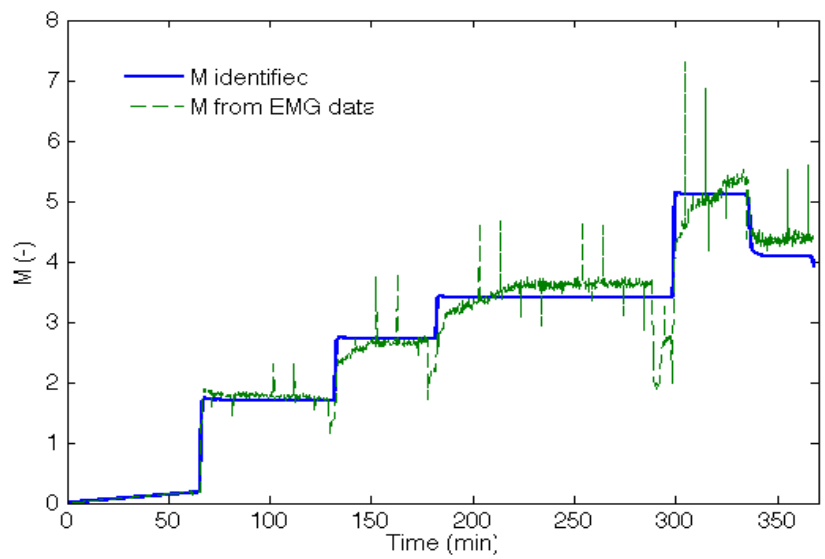

Fig. 4. Representation of the identified $M$ and the so-called measured $M$ 


\subsection{Extended Prediction Self-Adaptive Control}

In this paper, we apply the EPSAC (Extended Prediction Self-Adaptive Control) strategy described in detail in (De Keyser, 2003). The EPSAC-MPC (EPSAC - Model Predictive Control) is based on a generic process model:

$$
y(t)=x(t)+n(t)
$$

The disturbance $n(t)$ includes the effects in the measured output $y(t)$ which do not come from the model input $u(t)$ via the available model. These non-measurable disturbances have a stochastic character with non-zero average value, which can be modelled by a coloured noise process:

$$
n(t)=\left[C\left(q^{-1}\right) / D\left(q^{-1}\right)\right] \cdot e(t)
$$

with: $e(t)$ - uncorrelated (white) noise with zero mean value; $C\left(q^{-1}\right)$ and $D\left(q^{-1}\right)$ - monic polynomials in the backward shift operator $q^{-1}$ of orders $n_{c}$ and $n_{d}$. The disturbance filter $C\left(q^{-1}\right) / D\left(q^{-1}\right)$ is defined as a pure integrator, to ensure zero steady state error.

The relationship between $u(t)$ and $x(t)$ is given by the generic dynamic system model:

$$
x(t)=f[x(t-1), x(t-2), \cdots, u(t-1), u(t-2), \cdots]
$$

In our case the input applied to the patient, $u(t)$, is a vector containing the Propofol and Remifentanil delivery rates. The prediction model output is not represented by a nonlinear Hill curve, but by a linear approximation around the maintenance values (i.e. BIS values between $40 \%$ and $60 \%$ ) (Nascu et al, 2011):

$$
x(t)=m_{1} \cdot C_{e \operatorname{Pr} o p}\left(t-T_{d}\right)+m_{2} \cdot C_{e \operatorname{Re} m}\left(t-T_{d}\right)
$$

The process output is predicted at time instant $t$ over the prediction horizon after the time delay $T_{d}$, based on the measurements available at that moment and the future outputs of the control signal. The predicted values of the output are:

$$
y(t+k / t)=x(t+k / t)+n(t+k / t)
$$

Prediction of $x(t+k \mid t)$ and of $n(t+k \mid t)$ can be done respectively by recursion of the process model and by using filtering techniques on the noise model (11) (De Keyser, 2003).

In EPSAC for linear models, the future response is considered as being the cumulative result of two effects:

$$
y(t+k / t)=y_{\text {base }}(t+k / t)+y_{\text {opt }}(t+k / t)
$$

where $y_{\text {base }}(t+k / t)$ represents:

- $\quad$ effect of past control $\{u(t-1), u(t-2), \ldots\}$ (initial conditions at time $t$ );

- effect of a base future control scenario, called $u_{\text {base }}(t+k \mid t), \quad k \geq 0$, which is defined a priori; for linear systems the choice is irrelevant, a simple choice being $\left\{u_{\text {base }}(t+k \mid t) \equiv 0, \quad k \geq 0\right\}$;

- $\quad$ effect of future (predicted) disturbances $n(t+k \mid t)$. and $y_{\text {opt }}(t+k / t)$ represents:

- effect of the optimizing future control actions $\left\{\delta u(t \mid t), \quad \delta u(t+1 \mid t), \mathrm{K} \quad \delta u\left(t+N_{u}-1 \mid t\right)\right\} \quad$ with $\delta u(t+k \mid t)=u(t+k \mid t)-u_{\text {base }}(t+k \mid t)$. The design parameter $N_{u}$, called the control horizon (a well-known concept in MPC-literature), is considered in this paper equal to 1.

The controller output is obtained by minimizing:

$$
J(\mathbf{U})=\sum_{k=N_{1}}^{N_{2}}[r(t+k / t)-y(t+k / t)]^{2}
$$

where $r(t+k / t)$ is the desired reference trajectory. The detailed formulation is given in (De Keyser, 2003) together with a multivariable formulation.

\subsection{Simulation Results}

The simulation of the closed loop control performance is performed in the context of using the nonlinear patient simulator described in section 3.1. In the predictive control algorithm, the prediction model for the patient is a linear approximation of the full nonlinear model. This linear approximation has been previously described in (Nascu et al, 2011) and consists of the PKPD model from (1)-(2) with a linear approximation of the plane given by (3), i.e. (13) with $m_{1}=12.83$ and $m_{2}=7.73$. The control algorithm has a sampling period of 5 seconds, a prediction horizon of 20 samples.
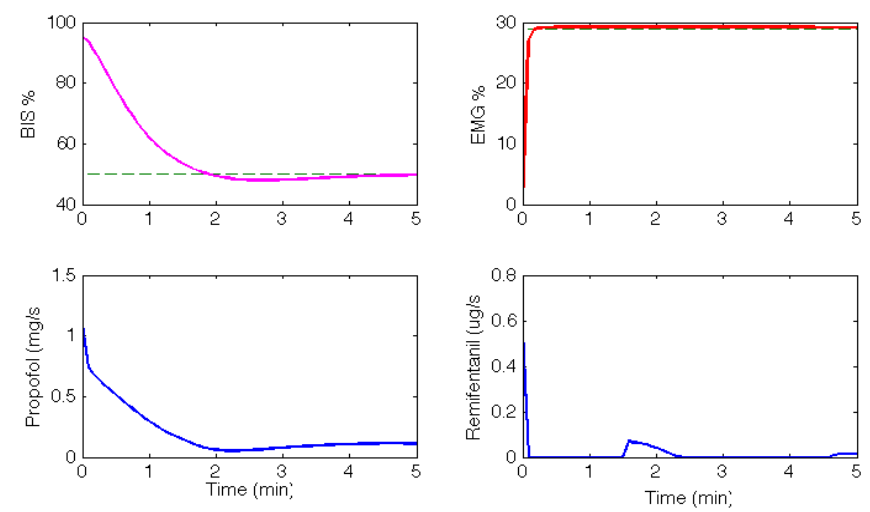

Fig. 5: Simulation test during the induction phase
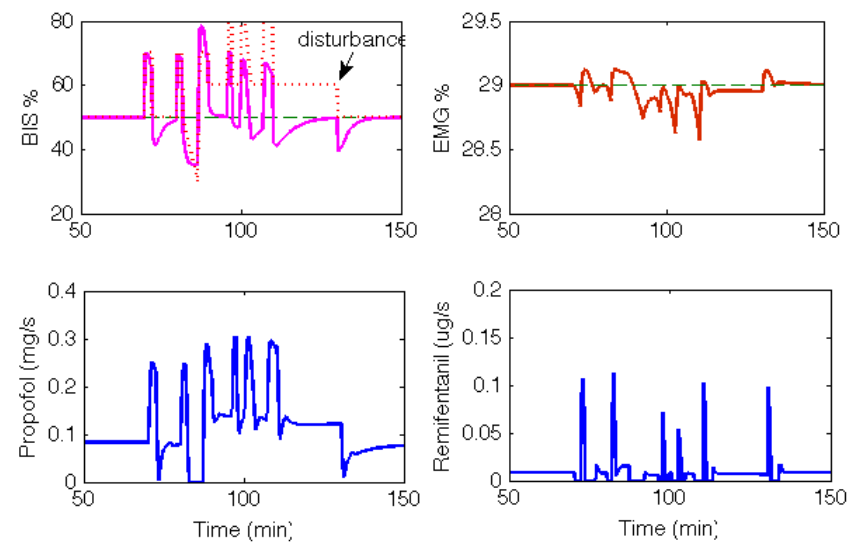

Fig. 6: Simulation test during the maintenance phase

Figure 5 depicts the simulation results during the induction phase. Bispectral index and EMG are controlled variables, at $50 \%$, respectively $29 \%$ reference values. The manipulated variables are Propofol and Remifentanil. Although fast, the controller brings the patient to the desired values without overshoot. Recall that in this case, there are significantly 
large differences between the nonlinear model of the patient simulator and the linear approximation of the prediction model.

For the maintenance phase, a signal with clinically realistic disturbances has been applied. This signal has been developed and introduced in (Yelneedi et al, 2009). Figure 6 depicts the results of the disturbance rejection test during maintenance phase, where one can also observe the disturbance signal applied into the control scheme. The performance of the controller is quite good and stable. However, some high-peaks are observed in the BIS signal output as a result of the high disturbance effect. These peaks can be minimized if an adaptive control strategy is introduced, resulting in a patient-individualized DOA regulation framework. However, the topic of such adaptive control scheme is the subject of another paper.

\section{CONCLUSIONS}

In this paper is presented a brief state of art regarding the multivariable formulation for controlling the depth of anaesthesia by means of two intravenously administrated drugs, i.e. Propofol and Remifentanil. In a feasibility study of determining a suitable variable to quantify analgesia levels in patients undergoing cardiac surgery, the Bispectral index and an electromyogram-based surrogate variable are proposed as the controlled variables. The study is carried on in the context of implementing a multivariable predictive control algorithm. The simulation results show that such a paradigm is feasible, although it does not guarantee perfect knowledge of the clinical analgesia level - in other words, the variable is not validated against typical evaluations of the pain levels (eg. Clinical scores). This means that the strategy is not yet ready for clinical practice. These results can be further improved if an adaptive control strategy is introduced, resulting in a patient-individualized DOA regulation framework. However, the topic of such adaptive control scheme is the subject of another paper.

\section{ACKNOWLEDGEMENT}

C.M. Ionescu is a post-doctoral fellow of Flanders Research Foundation (FWO).

\section{REFERENCES}

Absalom A., De Keyser R., Struys M.R.F., (2011) Closed loop anaesthesia: are we getting close to finding the Holy Grail?, AnesthAnalg,112(3), 516-518

Absalom AR, Mani V, De Smet T, Struys MM. (2009) Pharmacokinetic models for propofol-defining and illuminating the devil in the detail Br J Anaesth;103:26-37

Bouillon TW (2008) Hypnotic and Opioid Anesthetic Drug Interactions on the CNS, Focus on Response Surface Modelling.In: Schuttler J, Schwilden H, eds. Modern Anesthetics, Handbook of Experimental Pharmacology 182. Berlin Heidelberg: Springer-Verlag, 471-487

Caruso A, Bouillon TW, Schumacher PM, Zanderigo E, Morari M, (2009) Control of drug administration during monitored anaesthesia care, IEEE Trans Automation Science and Engineering, 6(2), 256-264

Da Silva M, Mendonca T, Wigren, T, (2011) Nonlinear adaptive control of neuromuscular blockade in anaesthesia, $50^{\text {th }}$ IEEE Conf on Decision and Control, joint with European Conf Control, Orlando, FL, 47-52

De Keyser R., (2003) Model Based Predictive Control for Linear Systems, UNESCO Encyclopaedia of Life Support Systems, Article contribution 6.43.16.1, Eolss Publishers Co Ltd, Oxford
Dumont G, Martinez A, Ansermino M, (2009) Robust control of depth of anaesthesia, Int J Adapt Control Signal Process, 23, 435-454

Hemmerling TM, Salhab E, Aoun G, Charabati S. Mathieu P, (2007) The "Analgoscore": a novel score to monitor intraoperative pain and its use for Remifentanil closed loop application, IEEE Proceedings Cat., ref nr 1-4244-0991-8/07, 1494-1499

Ionescu C. M., R. De Keyser, B.C. Torrico, T. De Smet, M. Struys, J.E. Normey-Rico, (2008) Robust Predictive Control Strategy Applied for Propofol Dosing using BIS as a Controlled Variable during Anaesthesia, IEEE Trans Biomed Eng, 55, 2161-2170

Kazama T, Ikeda K, Morita K, Sanjo Y, (1998) Awakening Propofol concentration with and without blood-effect site equilibration after short-term and long-term administration of Propofol and fentanyl anaesthesia, Anesthesiology, 88, 928-934

Liley D, Sinclair N, Lipping T, Heyse B, Vereecke E.M., Struys M, (2010) Propofol and Remifentanil differentially modulate frontal electroencephalographic activity, Anesthesiology, 113, 292-304

Liu N, Chazot T, Genty A, Landais A, Restoux A, McGee K, Laloe PA, Trillat B, Barvais L, Fischler M. (2011) Closed-loop coadministration of Propofol and Remifentanil guided by Bispectral index: a randomized multicenter study. Anesth Analg,112(3), 546-557

Mahfouf M, Nunes C., Linkes D, Peacock J, (2005) Modelling and multivariable control in anaesthesia using neural-fuzzy paradigms, Part II: closed-loop control of simultaneous administration of Propofol and Remifentanil, Artif Intell in Med, 35, 207-213

Milne S, Kenny G, Schraag S, (2003) Propofol sparing effect of Remifentanil using closed loop anaesthesia, Brit J Anaesthesia, 90(5), 623-629

Minto C. F., Schnider T. W., Short T.G., Gregg K. M., Gentilini A., Shafer S. L. (2000) Response surface model for anaesthetic drug interactions, Anesthesiology, 92, 1603-1616

Minto CF, TW Schnider, TD Egan, E Youngs, HJ Lemmens, PL Gambús, V Billard, JF Hoke, KH Moore, DJ Hermann, KT Muir, JW Mandema, SL Shafer, (1997a) Influence of age and gender on the pharmacokinetics and pharmacodynamics of Remifentanil. I. Model development, Anesthesiology, 86, 10-23

Minto CF, M White, N Morton, GN Kenny, (1997b) Pharmacokinetics and pharmacodynamics of Remifentanil. II Model application, Anesthesiology, 86, 24-33

Morley A, Derrick J, Mainland P, Lee BB, Short TG. (2000) Closed loop control of anaesthesia: an assessment of the bispectral index as the target of control. Anaesthesia, 55, 953-959

Nascu I(a), Ionescu CM, Nascu I, De Keyser R, (2011), "Evaluation of three protocols for automatic DOA regulation using Propofol and Remifentanil “, The 9th IEEE International Conference on Control \& Automation 2011, Santiago, Chile

Pattinson K, Governo R, MacIntosh B, Russell E, Corfield D, Tracey I, Wise $\mathrm{R}$, (2009) Opioids depress cortical centers responsible for the volitional control of respiration, J Neurosc, 29(25), 8177-8186

Schnider TW, CF Minto, SL Shafer, PL Gambús, C Andresen, DB Goodale, EJ Youngs, (1999) The influence of age on Propofol pharmacodynamics, Anesthesiology, 90, 1502-1516

Schubert A, Janda M, Simanski O, Bajorat J, Pohl B, Hofmockel R, Lapme $\mathrm{B}$, (2008) A fuzzy system for regulation of the analgesic Remifentanil during general anaesthesia, $16^{\text {th }}$ Med Conf Contr and Autom, Ajaccio, France, 1634-1639

Simanski O, Janda M, Schuibert A, Bajorat J, Hofmockel R, Lampe B, (2009) Progress of automatic drug delivery in anaesthesia - the 'Rostock' assistant system for anaesthesia control (RAN), Int J Adapt Control Signal Process, 23, 504-521

Struys M., H. Vereecke, A. Moerman, Jensen EW, Verhaeghen D, De Neve N, Dumortier FJ, Mortier EP, (2003) Ability of the bispectral index, autoregressive modeling with exogenous input-derived auditory evoked potentials and predicted Propofol concentrations to measure patient responsiveness during anaesthesia, Anesthesiology, 99, 802-812

Yelneedi S., L. Samavedham, G.P. Rangaiah, (2010), Automatic regulation of anaesthesia by simultaneous administration of two anaesthetic drugs using model predictive control, IFMBE Proceedings, 14(1), 82-86

Yelneedi S., L. Samavedham, G.P. Rangaiah, (2009) Advanced control strategies for the regulation of hypnosis with Propofol, Ind Eng Chem Res, 48: 3880-3897

Zikov T, Bibian, Dumont G, (2006) Quantifying cortical activity during general anaesthesia using wavelet analysis, IEEE Trans Biomed Eng, 53(4), 617-632 